Research Article

\title{
Elliptic Equation of Plastic Area Boundary around the Circular Laneway in Nonuniform Stress Field
}

\author{
Xiaofei Guo $\mathbb{i}$ and Linfeng Guo \\ School of Energy and Mining Engineering, China University of Mining and Technology(Beijing), Beijing 100083, China \\ Correspondence should be addressed to Linfeng Guo; guolinfeng@student.cumtb.edu.cn
}

Received 19 August 2020; Revised 10 September 2020; Accepted 11 October 2020; Published 2 December 2020

Academic Editor: Bangyou Jiang

Copyright (C) 2020 Xiaofei Guo and Linfeng Guo. This is an open access article distributed under the Creative Commons Attribution License, which permits unrestricted use, distribution, and reproduction in any medium, provided the original work is properly cited.

In order to obtain the analytical solution of the plastic area boundary of circular laneway surrounding rock in nonuniform stress field, we studied the evolution of the plastic area shapes of the circular laneway surrounding rock from circular to elliptical and derived the analytical solutions of the boundary radii in the elliptical shape. The results show that (1) with the increase of the confining pressure ratio from 1, the major axis radius of the plastic area increases gradually, the minor axis radius decreases gradually, and the shape of the plastic area gradually evolves from circular to elliptical; (2) on the basis of the Mohr-Coulomb strength criterion, the analytical expressions of major axis and minor axis radii of the elliptical plastic area are derived, and the elliptic equation of the plastic area boundary of circular laneway in nonuniform stress field is established; and (3) the confining pressure ratio is the key factor affecting the shape of the plastic area. When the confining pressure ratio is less than 1.6, the plastic area of the circular laneway surrounding rock is elliptical, and the elliptic boundary equation is applicable. When the confining pressure ratio is greater than 1.6 , the plastic area is butterfly shaped, and the elliptic boundary equation is no longer applicable.

\section{Introduction}

In underground mining, the laneway passes through the complex geological environment, and roof fall, floor heave, and other laneway disasters have been a major threat to the safety production of coal mines [1-3]. Elastoplastic theory is an effective theoretical means to analyze the damage degree, form, and laws of surrounding rock [4-6]. At present, the research of the uniform confining pressure model is more mature, and the plastic circle radius calculated by the Fenner and Kastner formulas has been widely used in theory and practice $[7,8]$. However, in the actual underground environment, the stress field around the laneway is complex, especially affected by the tectonic stress and mining stress, and the stress field is often nonuniform $[9,10]$. At this time, the results obtained by the Fenner and Kastner formulas deviate greatly from the actual situation [11].

On the elastic-plastic analysis of laneway surrounding rock in nonuniform stress field, $\mathrm{Yu}$ et al. put forward the implicit equation of the circular laneway plastic area boundary [12]. After that, it has been further developed [13]. Then, Zhao et al. found that the shapes of the laneway plastic area would appear as elliptical and butterfly shapes in nonuniform stress field [14]. $\mathrm{Ma}$ and $\mathrm{Li}$ and Guo et al. introduced deviator stress to reveal the mechanical mechanism of the noncircular plastic area $[15,16]$. Guo et al. studied the general shapes of the circular laneway plastic area in nonuniform stress field and gave the mathematical definitions of the different shapes $[17,18]$.

The shape and range of the laneway plastic area in nonuniform stress field is of great significance to study the stability and control of surrounding rock. However, so far, there is still no analytical solution of the boundary radius of the laneway plastic area in the nonuniform stress field, which brings great inconvenience to the research work. In this paper, we studied the evolution of the circular laneway plastic area shapes from circular to elliptical and derived the analytical solutions of the boundary radii in the elliptical shape. 


\section{Evolution and Distribution Characteristics of the Elliptical Plastic Area}

2.1. Force Model and Boundary Conditions. The plane strain model of the circular laneway is often used to study the elastoplastic problems of the underground laneway surrounding rock under the condition of existing mathematical and mechanical methods. So, any section of the laneway can be taken as its representative in the infinite length. Considering the nonuniform distribution characteristics of complex stress fields such as underground tectonic stress and mining stress, we established the stress model of laneway surrounding rock in nonuniform stress field, as illustrated in Figure 1 . In the figure, $R$ and $\theta$ are the polar coordinates, and $a$ is the laneway radius. The complex stress environment around the laneway is represented by the regional principal stress field, where $P_{1}$ is the maximum confining pressure and
$P_{3}$ is the minimum confining pressure. To reflect the uneven distribution of regional stress field, the ratio of maximum to minimum confining pressure is defined as the confining pressure ratio, expressed as formula (1). The nonuniform distribution characteristics of complex stress field are represented by the size, direction, and ratio of confining pressure in this model.

$$
\eta=\frac{P_{1}}{P_{3}}
$$

According to the Mohr-Coulomb strength theory and the stress solutions around a circular hole in elasticity, the boundary equation of the plastic area surrounding the circular laneway in nonuniform stress field was deduced [12-14]. We can study the shape of plastic area according to the boundary equation. The expression is as follows:

$$
\begin{aligned}
& 9(1-\eta)^{2}\left(\frac{a}{r}\right)^{8}+\left[-12(1-\eta)^{2}+6(1-\eta) \cos 2 \theta\right]\left(\frac{a}{r}\right)^{6}+\left[\begin{array}{c}
10(1-\eta)^{2} \cos ^{2} 2 \theta-4(1-\eta)^{2} \sin ^{2} \varphi \cos ^{2} 2 \theta \\
-2(1-\eta)^{2} \sin ^{2} 2 \theta-4\left(1-\eta^{2}\right) \cos 2 \theta+(1+\eta)^{2}
\end{array}\right]\left(\frac{a}{r}\right)^{4} \\
& \quad+\left[\begin{array}{c}
-4(1-\eta)^{2} \cos 4 \theta+2\left(1-\eta^{2}\right) \cos 2 \theta \\
-4\left(1-\eta^{2}\right) \sin ^{2} \varphi \cos 2 \theta-\frac{4 C(\eta-1) \sin 2 \varphi \cos 2 \theta}{P_{3}}
\end{array}\right]\left(\frac{a}{r}\right)^{2}+\left[(1-\eta)^{2}-\sin ^{2} \varphi\left(1+\eta+\frac{2 C \cos \varphi}{P_{3} \sin \varphi}\right)^{2}\right]=0
\end{aligned}
$$

where $\varphi$ and $C$ are the rock internal friction angle and cohesion.

2.2. Evolution of the Elliptical Plastic Area. According to the implicit boundary equation, we obtained the evolution of the elliptical plastic area surrounding the circular laneway. Under certain conditions of surrounding rock lithology, the confining pressure ratio determines the shape of the laneway surrounding rock plastic area. When $\eta=1$, Figure 2 is the uniform confining pressure stress model, boundary equation (1) is the circular equation, and the plastic area of the laneway surrounding rock is circular. With the confining pressure ratio increased from 1 , the transverse axis radius of the plastic area decreases gradually, the longitudinal axis radius increases gradually, the boundary of the plastic area is smooth transition, and the plastic area is similar to ellipse shape. With the increase of the confining pressure ratio, the scope of the plastic area expands and elliptical feature is gradually obvious, as shown in Figure 1.

When $\eta \neq 1$, the transverse radius of the plastic area is the smallest and the longitudinal radius is the largest, and the shape of plastic area is similar to ellipse. We define the shape of the plastic area with the maximum radius on the longitudinal axis and the minimum radius on the transverse as the elliptical shape. The maximum radius on the longitudinal axis is defined as the major axis radius, expressed by $R_{\text {maj; }}$ the minimum radius on the transverse axis is defined as the major axis radius, expressed by $R_{\text {min. }}$. Elliptical distribution and special radii are shown in Figure 3. If the analytic formulas of the special radii were found, we would get the boundary radius of the plastic area at any position according to the elliptic equation.

\section{Elliptic Equation of Plastic Area Boundary}

3.1. Analytic Formula of Major Axis Radius. According to the elliptical shaped plastic area, by substituting $\theta=\pi / 2$ into the solution of stress around a circular hole in elasticity $[19,20]$, the solution of stress around a circular laneway on the vertical axis can be obtained as follows:

$$
\left\{\begin{array}{l}
\sigma_{\mathrm{yr}}=\frac{(1+\eta) P_{3}}{2}\left(1-\frac{a^{2}}{r^{2}}\right)-\frac{(\eta-1) P_{3}}{2}\left(1-4 \frac{a^{2}}{r^{2}}+3 \frac{a^{4}}{r^{4}}\right) \\
\sigma_{\mathrm{y} \theta}=\frac{(1+\eta) P_{3}}{2}\left(1+\frac{a^{2}}{r^{2}}\right)+\frac{(\eta-1) P_{3}}{2}\left(1+3 \frac{a^{4}}{r^{4}}\right) \\
\tau_{\mathrm{yr} \theta}=0
\end{array}\right.
$$

where $\sigma_{\mathrm{y} \theta}$ is the tangential stress, $\sigma_{\mathrm{yr}}$ is the radial stress, and $\tau_{\mathrm{yr} \theta}$ is the shearing stress of every point on the vertical axis.

The Mohr-Coulomb strength theory is applied to analyze the elastoplasticity of surrounding rock in this study. The maximum principal stress and minimum 


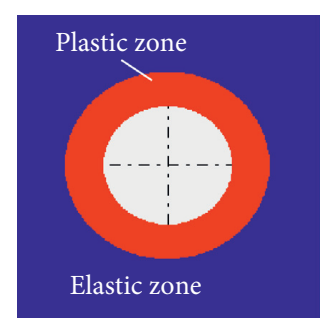

(a)

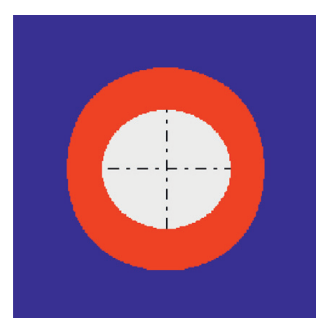

(b)

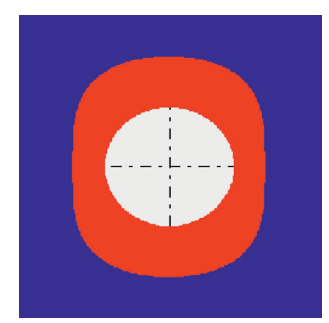

(c)

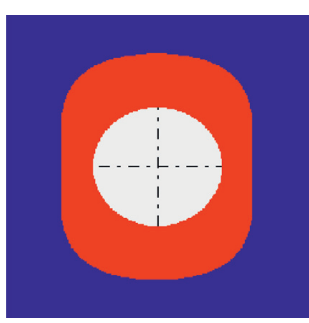

(d)

FIGURE 1: Evolution of the elliptical plastic area in nonuniform stress field $\left(a=2.5 \mathrm{~m} ; C=3 \mathrm{MPa} ; \varphi=25^{\circ} ; P_{3}=20 \mathrm{MPa}\right)$. (a) $\eta=1$. (b) $\eta=1.2$. (c) $\eta=1.4$. (d) $\eta=1.6$.

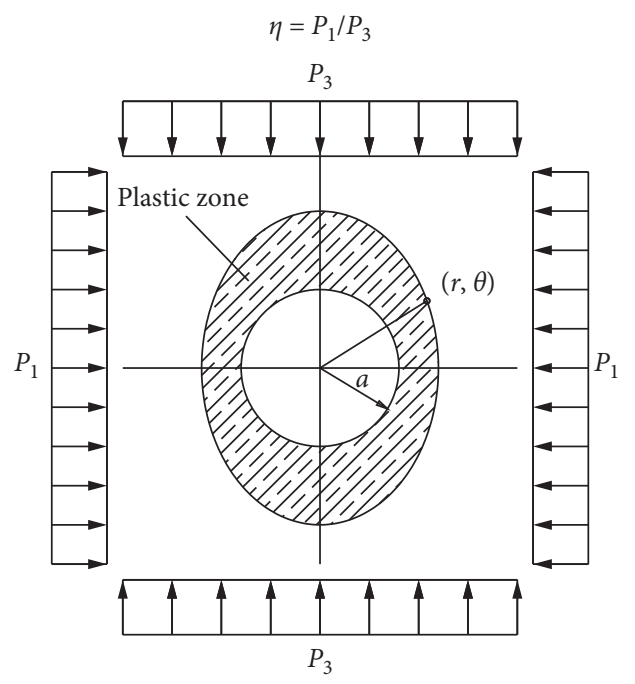

FIGURE 2: Circular laneway force model in nonuniform stress field. $a$ : laneway radius; $r, \theta$ : polar coordinates of any point on the boundary; $P_{1}$ : maximum confining pressure; $P_{3}$ : minimum confining pressure.

principal stress on the vertical axis are shown as follows $[19,20]$ :

$$
\left\{\begin{array}{l}
\sigma_{1}=\frac{\sigma_{\mathrm{yr}}+\sigma_{\mathrm{y} \theta}}{2}+\frac{1}{2} \sqrt{\left(\sigma_{\mathrm{yr}}-\sigma_{y \theta}\right)^{2}+4 \tau_{\mathrm{yr} \theta}^{2}}, \\
\sigma_{3}=\frac{\sigma_{\mathrm{yr}}+\sigma_{\mathrm{y} \theta}}{2}-\frac{1}{2} \sqrt{\left(\sigma_{r}-\sigma_{\theta}\right)^{2}+4 \tau_{\mathrm{yr} \theta}^{2}},
\end{array}\right.
$$

where $\sigma_{1}$ is the maximum principal stress and $\sigma_{3}$ is the minimum principal stress.

The difference between radial stress and tangential stress in formula (3) leads to the following formula:

$$
\sigma_{\mathrm{yr}}-\sigma_{\mathrm{y} \theta}=-(\eta+1) \frac{a^{2}}{r^{2}} P_{3}-(\eta-1) P_{3}\left(3 \frac{a^{4}}{r^{4}}-2 \frac{a^{2}}{r^{2}}+1\right)<0 .
$$

Then, formula (4) can be transformed as follows:

$$
\left\{\begin{array}{l}
\sigma_{1}=\sigma_{\mathrm{y} \theta} \\
\sigma_{3}=\sigma_{\mathrm{yr}}
\end{array}\right.
$$

Based on the Mohr-Coulomb strength theory, the following condition needs to be satisfied at the elastic-plastic interface on the vertical axis:

$$
\sigma_{y \theta}=2 C \frac{\cos \varphi}{1-\sin \varphi}+\frac{1+\sin \varphi}{1-\sin \varphi} \sigma_{\mathrm{yr}},
$$

where $C$ and $\varphi$ are the rock cohesion and internal friction angle. Furthermore, the plastic area boundary equation on the vertical axis is expressed as follows:

$$
a_{1} y^{2}+b_{1} y+c_{1}=0
$$

where $Y=a^{2} / r^{2} ; a_{1}=(6(\eta-1)) /(1-\sin \varphi) ; b_{1}=(\eta+1)-$ $((3 \eta-5)(1+\sin \varphi) /(1-\sin \varphi)) ;$ and $c_{1}=2 \eta-(4 C \cos \varphi /$ $\left.P_{3}(1-\sin \varphi)\right)-(2(1+\sin \varphi) /(1-\sin \varphi))$.

The solutions of equation (8) are as follows:

$$
\begin{aligned}
& y_{1}=\frac{-b_{1}+\sqrt{b_{1}^{2}-4 a_{1} c_{1}}}{2 a_{1}}, \\
& y_{2}=\frac{-b_{1}-\sqrt{b_{1}^{2}-4 a_{1} c_{1}}}{2 a_{1}} .
\end{aligned}
$$

Before the laneway is excavated, the surrounding rock is in an elastic state, while local surrounding rock around the laneway is in a plastic state after excavation. Based on the Mohr-Coulomb failure criterion, the stress around the laneway before excavation must meet the following conditions:

$$
\eta<2 C \frac{\cos \varphi}{(1-\sin \varphi) P_{3}}+\frac{1+\sin \varphi}{1-\sin \varphi} .
$$

Therefore, $a_{1}>0$ and $c_{1}<0$, and $-b_{1}-\sqrt{b_{1}^{2}-4 a_{1} c_{1}}<0$ and $y_{2}<0, y_{2}$ is a meaningless solution. The analytical formula for the major axis radius of the elliptical plastic area is expressed as follows:

$$
R_{\mathrm{maj}}=\frac{a}{\sqrt{y_{1}}}
$$

where $y_{1}$ is the analytic expression about the stresses (the maximum and minimum principal stresses) and the lithology of the surrounding rock (rock cohesion and internal friction angle), so the major axis radius can be solved when these parameters are known. 


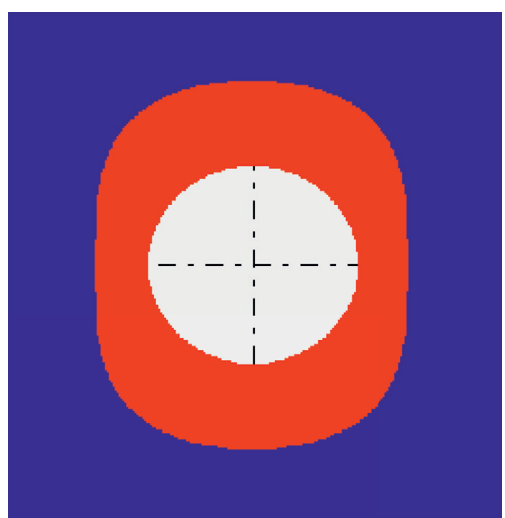

FIGURE 3: Elliptical distribution and special radii. (a) $\eta=1$.

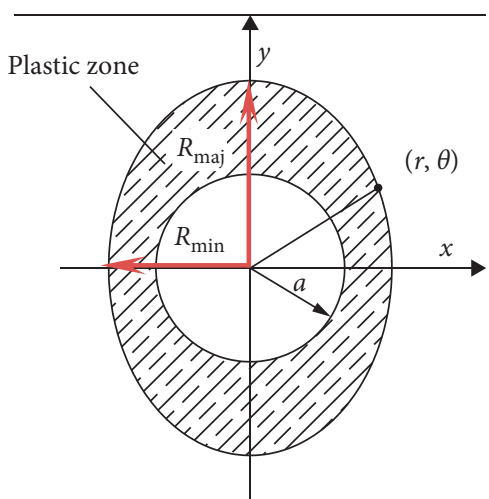

(b) $\eta=1.2$. (c) $\eta=1.4$. (d) $\eta=1.6$.
3.2. Analytic Formula of Minor Axis Radius. Based on the method of solving the major axis radius, by substituting $\theta=0$ into the solution of stress around a circular hole in elasticity, the solution of stress around a circular laneway on the abscissa axis can be obtained as follows:

$$
\left\{\begin{array}{l}
\sigma_{\mathrm{xr}}=\frac{(\eta+1) P_{3}}{2}\left(1-\frac{a^{2}}{r^{2}}\right)+\frac{(\eta-1) P_{3}}{2}\left(1-4 \frac{a^{2}}{r^{2}}+3 \frac{a^{4}}{r^{4}}\right), \\
\sigma_{x \theta}=\frac{(\eta+1) P_{3}}{2}\left(1+\frac{a^{2}}{r^{2}}\right)-\frac{(\eta-1) P_{3}}{2}\left(1+3 \frac{a^{4}}{r^{4}}\right), \\
\tau_{\mathrm{x} r \theta}=0
\end{array}\right.
$$

where $\sigma_{x r}$ is the radial stress, $\sigma_{x \theta}$ is the tangential stress, and $\tau_{\mathrm{xr} \theta}$ is the shearing stress.

The difference between radial stress and tangential stress in formula (12) leads to the following formula:

$$
\sigma_{\mathrm{x} r}-\sigma_{x \theta}=-(\eta+1) P_{3} \frac{a^{2}}{r^{2}}+(\eta-1) P\left(3 \frac{a^{4}}{r^{4}}-2 \frac{a^{2}}{r^{2}}+1\right) \text {. }
$$

Because the sign of the difference between radial and tangential stresses cannot be judged directly, assuming $x=a^{2} / r^{2}$, formula (13) is expressed as follows:

$$
P(x)=3(\eta-1) P_{3} x^{2}+(1-3 \eta) P_{3} x+(\eta-1) P_{3} .
$$

The expression of two roots is obtained by solving equation $P(x)=0$.

$$
\begin{aligned}
& x_{p 1}=\frac{(3 \eta-1)-\sqrt{-3 \eta^{2}+18 \eta-11}}{6(\eta-1)}, \\
& x_{p 2}=\frac{(3 \eta-1)+\sqrt{-3 \eta^{2}+18 \eta-11}}{6(\eta-1)} .
\end{aligned}
$$

When $x$ meets the conditions of $x<x_{p 1}$ or $x>x_{p 2}$, $P(x)>0 P(x)>0$. When $x$ meets the conditions of $x_{p 1}<x<x_{p 2}, P(x)<0$.
(1) When $P(x)>0, \sigma_{x r}$ is the maximum principal stress and $\sigma_{x \theta}$ is the minimum principal stress. Substituting stresses into the Mohr-Coulomb strength theory, the plastic area boundary equation on the abscissa axis can be expressed as follows:

$$
a_{2} x^{2}+b_{2} x+c_{2}=0
$$

where $a_{2}=(6(\eta-1) /(1-\sin \varphi)) ; \quad b_{2}=(3-5 \eta)-$ $((\eta+1)(1+\sin \varphi) /(1-\sin \varphi)) ;$ and $c_{2}=2 \eta-(4 C$ $\left.\cos \varphi / P_{3}(1-\sin \varphi)\right)-2(1+\sin \varphi) /(1-\sin \varphi)$.

The roots of equation (16) are expressed as follows:

$$
\begin{aligned}
& x_{1}=\frac{-b_{2}+\sqrt{b_{2}^{2}-4 a_{2} c_{2}}}{2 a_{2}}, \\
& x_{2}=\frac{-b_{2}-\sqrt{b_{2}^{2}-4 a_{2} c_{2}}}{2 a_{2}},
\end{aligned}
$$

where $a_{2}>0, c_{2}<0,-b_{2}-\sqrt{b_{2}^{2}-4 a_{2} c_{2}}<0$, and $x_{2}<0 . x_{2}$ is a meaningless solution. When $x_{1}$ meets the condition of $x_{1}<x_{p 1}$ or $x_{1}>x_{p 2}$, it will be a useful solution.

(2) When $P(x)<0, \sigma_{x \theta}$ is the maximum principal stress and $\sigma_{x r}$ is the minimum principal stress. Substituting stresses into the Mohr-Coulomb strength theory, the plastic area boundary equation on the abscissa axis can be expressed as follows:

$$
a_{3} x^{2}+b_{3} x+c_{3}=0
$$

where $\quad a_{3}=(6(1-\eta) /(1-\sin \varphi)) ; \quad b_{3}=(\eta+1)-$ $((3-5 \eta)(1+\sin \varphi) /(1-\sin \varphi))$; and $c_{3}=2 \eta-(4 C$ $\left.\cos \varphi / P_{3}(1-\sin \varphi)\right)-(2 \eta(1+\sin \varphi) /(1-\sin \varphi))$.

The roots of equation (18) are expressed as follows:

$$
\begin{aligned}
& x_{3}=\frac{-b_{3}+\sqrt{b_{3}^{2}-4 a_{3} c_{3}}}{2 a_{3}}, \\
& x_{4}=\frac{-b_{3}-\sqrt{b_{3}^{2}-4 a_{3} c_{3}}}{2 a_{3}} .
\end{aligned}
$$


When $x_{3}$ and $x_{4}$ meet the condition $x_{p 1}<x_{3}<x_{4}<x_{p 2}$, they will be useful solutions.

At least one of the three roots $\left(x_{1}, x_{3}\right.$, and $\left.x_{4}\right)$ is a useful root, and the minimum of the roots should be used. The analytical formula for the minor axis radius of the elliptical plastic area is expressed as follows:

$$
R_{\min }=\frac{a}{\sqrt{\min \left(x_{1}, x_{3}, x_{4}\right)}}
$$

where $x_{1}, x_{3}$, and $x_{4}$ are the analytical formulas about the stresses and the lithology of the surrounding rock, so the minor axis radius will be obtained when the parameters are known.

3.3. Elliptic Equation. With the confining pressure ratio increased from 1, the minor axis radius of the plastic area decreases gradually, the major axis radius increases gradually, the boundary of the plastic area is smooth transition, and the plastic area shows elliptical shape. The analytic expressions of the major and the minor axis radii of the ellipse are obtained. We can get the boundary radius of the plastic area at any position according to the elliptic equation. The elliptic equation of plastic area boundary around the circular laneway in nonuniform stress field is expressed as follows:

$$
R_{\theta}=\sqrt{R_{\mathrm{maj}}^{2} \sin ^{2} \theta+R_{\mathrm{min}}^{2} \cos ^{2} \theta},
$$

where $R_{\theta}$ is the boundary radius of the elliptical plastic area corresponding to polar angle $\theta . R_{\mathrm{maj}}$ and $R_{\mathrm{min}}$ are the analytical formulas about the stresses and the lithology of the surrounding rock, as shown in formulas (11) and (20), and they will be solved when the parameters are obtained. The special radii and boundary radium of the elliptical plastic area are shown in Figure 4.

3.4. Comparison with Existing Theoretical Formulas. On the basis of the Mohr-Coulomb failure criterion and the stress solutions around the circular laneway in elasticity, the implicit boundary equation of the plastic area surrounding the circular laneway in nonuniform stress was obtained [12-14]. So far, it has been widely used in in many fields of coal mine, such as the rock burst in the laneway $[16,21,22]$, the coal and gas outburst in the laneway [21], the roof falling in the laneway $[23,24]$, the large deformation in the laneway [14], and the permeability enhancement of coal seam $[25,26]$. However, through the implicit boundary equation, we can only get the numerical solutions of the plastic area boundary radii with the help of relevant software. The analytical solutions of the plastic area boundary radius can be obtained by the elliptic equation. In this study, we discuss the reliabilities of the solutions of elliptic equation by comparing with the results of implicit boundary equation, as shown in Figure 5 .

In Figure 5, the coal mechanical parameters $(C=3 \mathrm{MPa}$ and $\varphi=25^{\circ}$ ) are referred [17]; the circular laneway radius is $2.5 \mathrm{~m}$; the minimum confining pressure is fixed $\left(P_{3}=20 \mathrm{MPa}\right)$; and the different force models are set by

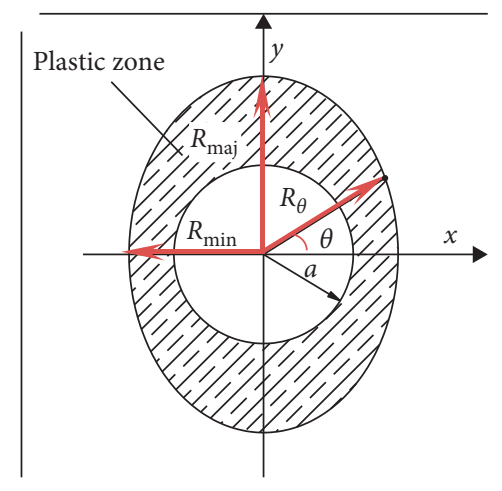

FIGURE 4: Special radii and boundary radium of the elliptical plastic area.

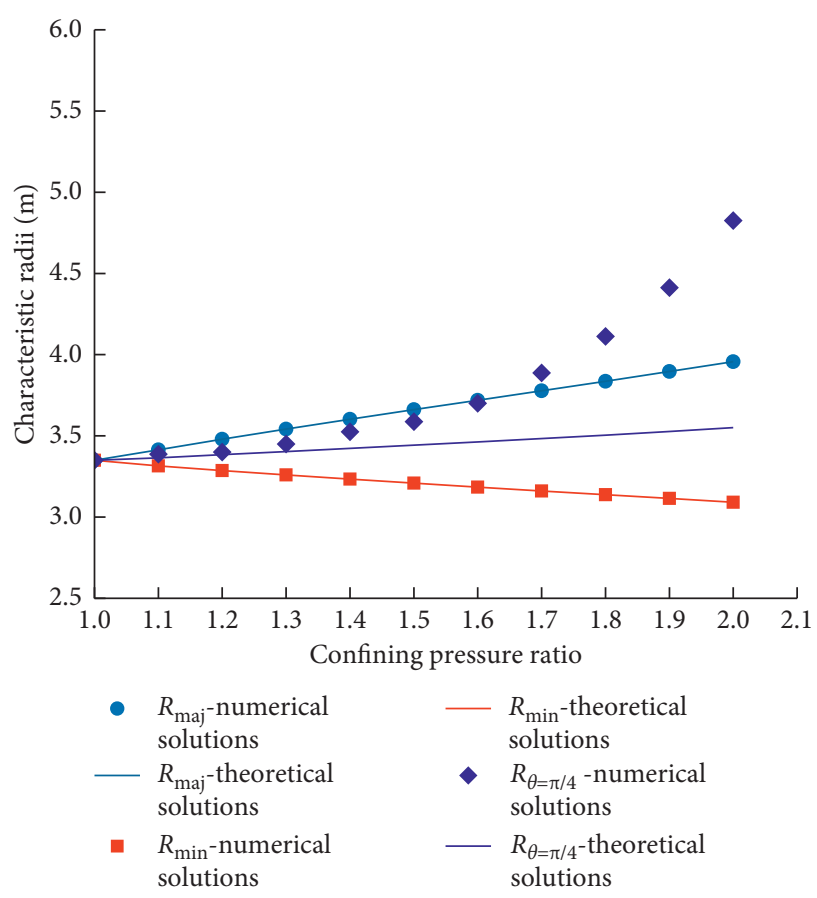

FIgURE 5: Error analysis for analytical solutions of special radii $\left(a=2.5 \mathrm{~m} ; C=3 \mathrm{MPa} ; \varphi=25^{\circ} ; P_{3}=20 \mathrm{MPa}\right)$.

changing the maximum confining pressure. The numerical solutions of boundary radii can be obtained by solving the implicit boundary equation with MATLAB. The results show that the analytical solutions of the major and minor axis radii are in good agreement with the numerical solutions and that the analytical formula of special radii is reliable. There is a certain deviation between the numerical solutions and the analytical solutions for the medial radius $(\theta=\pi / 4)$; when the confining pressure ratio is less than 1.6 , the deviation is small; when the confining pressure ratio is greater than 1.6, the deviation gradually increases with the increase of the confining pressure ratio. So, we can conclude that the elliptical plastic area defined in this paper is not a standard ellipse. And there is an error in solving the boundary radius beyond the special radii by using the elliptic equation of the plastic area boundary. In the following, the applicable conditions of elliptic equation are analyzed in detail. 


\section{Analysis of Influence Factors on Special Radii and Shapes of Plastic Area}

The shape of the elliptical plastic area is determined by the major and minor axes radii. In order to explore the influence of various factors on the laneway plastic area shape, it is necessary to study the relationship between the special radii and parameters. From the analytical formula, we can know that the main factors affecting the special radii are laneway radius, confining pressure ratio, internal friction angle, cohesion, and minimum confining pressure.

4.1. Laneway Radius. According to formulas (11), (20), and (21), it can be concluded that the boundary radius of the plastic area is proportional to the laneway radius. Figure 6 shows the relationship between the special radii and the laneway radius. In Figure 6, the coal mechanical parameters are referred $\left(C=3 \mathrm{MPa}\right.$ and $\varphi=25^{\circ}$ ), the confining pressure ratio is set as $1.4(\eta=1.4)$, and the minimum confining pressure is set as the fixed value $\left(P_{3}=20 \mathrm{MPa}\right)$. With the laneway radium increasing gradually from $1 \mathrm{~m}$, the major and minor radii increase linearly, and the range of the plastic area will increase proportionally.

4.2. Confining Pressure Ratio. Figure 7 shows the relationship between the special radii and the confining pressure ratio. In Figure 7, the rock mechanics parameters are set as $C=3 \mathrm{MPa}$ and $\varphi=25^{\circ}$, the laneway radium is set as $2.5 \mathrm{~m}$, and the minimum confining pressure is set as the fixed value $\left(P_{3}=20 \mathrm{MPa}\right)$. When the confining pressure ratio is 1 , the major axis radius and minor axis radius of the plastic area are equal, and the plastic area is in a circular shape. With the confining pressure ratio increasing from 1, the major axis radium increases gradually, while the minor axis radium decreases linearly; the difference between the radius of major axis and minor axis increases, and the elliptical shape of the plastic area tends to be obvious gradually (the larger the difference between major axis radium and minor axis radium, the more obvious the ellipse feature). So, the confining pressure ratio is the key factor affecting the shape of the plastic area.

4.3. Cohesion. In Figure 8, the laneway radium is taken as $2.5 \mathrm{~m}$, the rock internal friction angle is taken as $25^{\circ}$, the confining pressure ratio is taken as $1.4(\eta=1.4)$, and the minimum confining pressure is set as the fixed value $\left(P_{3}=20 \mathrm{MPa}\right)$. As can be seen from the relationship between special radii and cohesion, there is a negative correlation between them. With the increase of rock cohesion, the range of the plastic area decreases gradually. When the confining pressure ratio is constant, the two characteristic radius curves are approximately parallel. The influence of rock cohesion on the difference of the major axis radius and the minor axis radium is small. It can be concluded that the change of rock cohesion is significant in the range of the plastic area but has little effect on the shape.

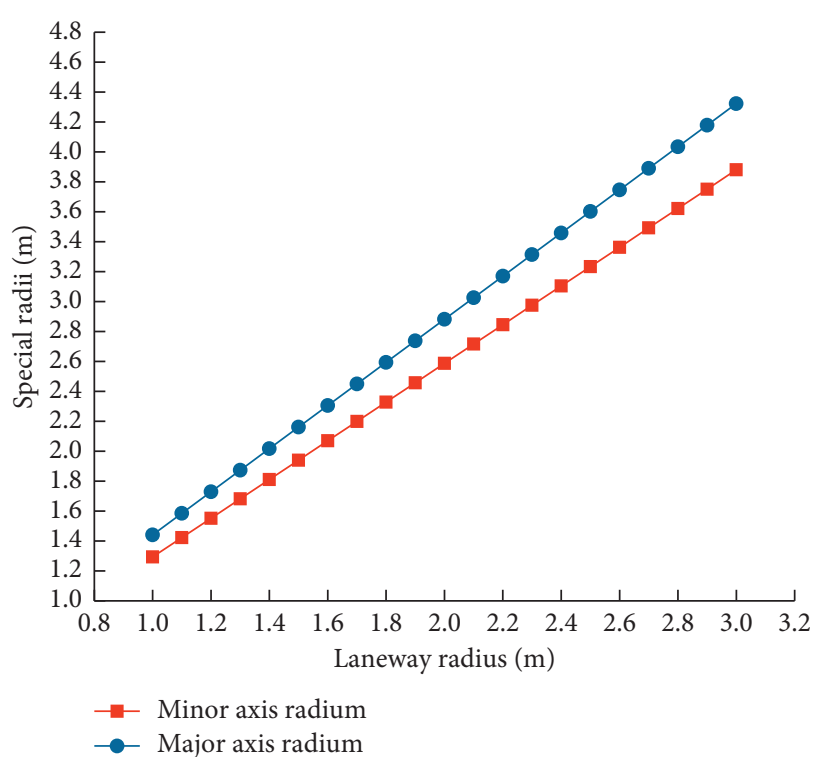

Figure 6: The relationship between special radii and laneway radius.

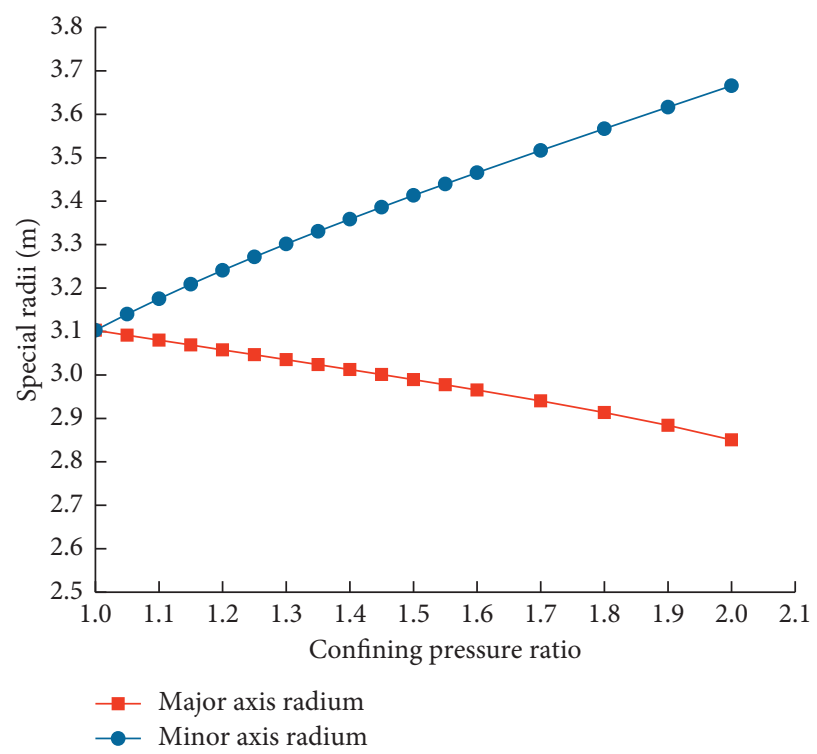

FIgURE 7: The relationship between special radii and confining pressure ratio.

4.4. Internal Friction Angle. In Figure 9, the laneway radium is set as $2.5 \mathrm{~m}$, the rock cohesion is taken as $3 \mathrm{MPa}$, the confining pressure ratio is set as $1.4(\eta=1.4)$, and the minimum confining pressure is taken as the fixed value $\left(P_{3}=20 \mathrm{MPa}\right)$. From the relationship between special radii and internal friction angle, we can see that there is a negative correlation between them. With the increase of the internal friction angle, the difference between the major axis radius and minor axis radium decreases, and the elliptical feature of the plastic area gradually weakens. The internal friction angle is another important factor affecting the shape of plastic area, which not only controls the range of the plastic area but also affects the shape characteristics. 


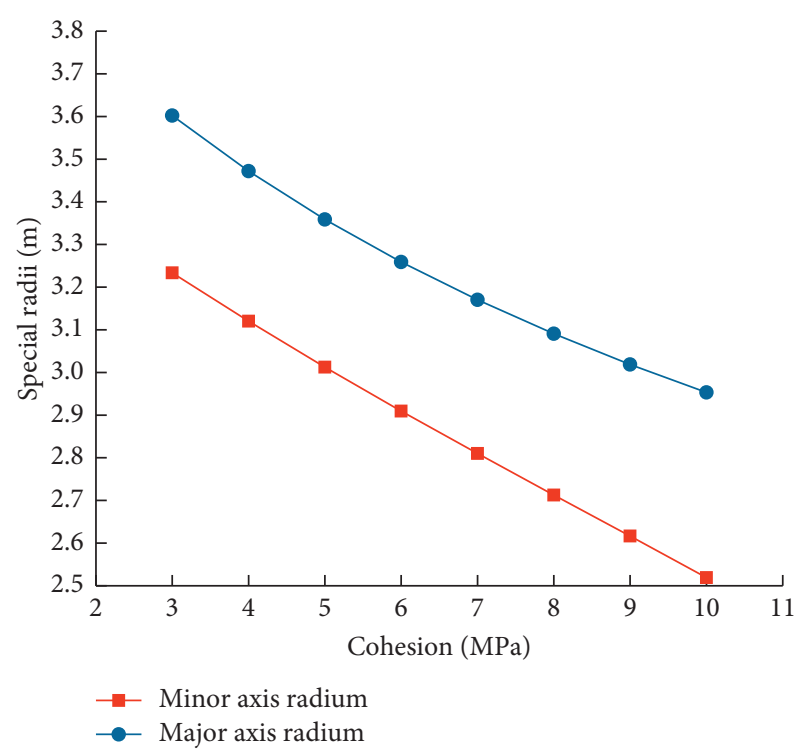

Figure 8: The relationship between special radii and cohesion.

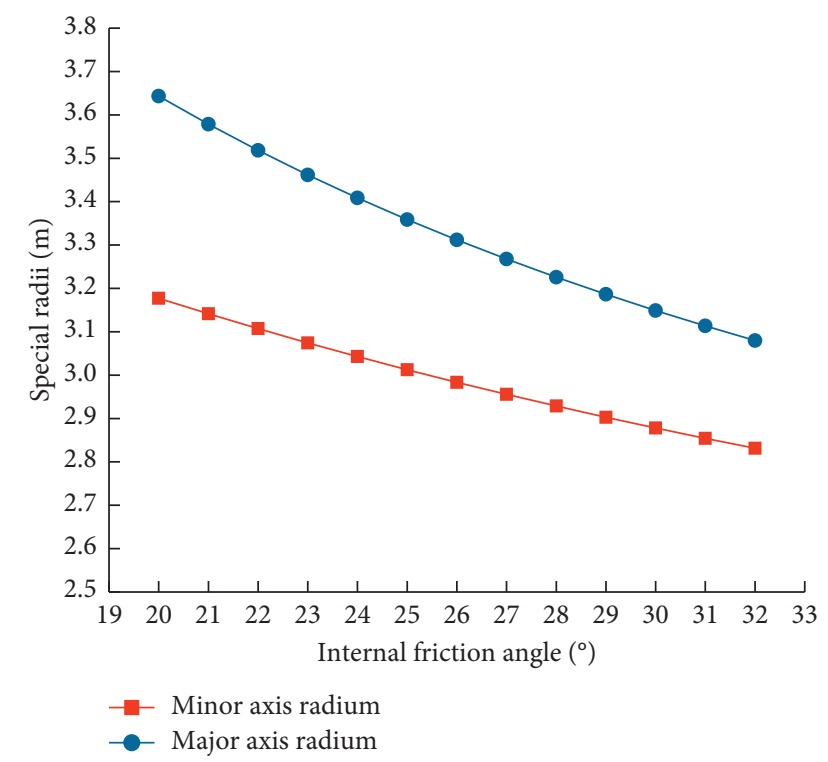

FIGURE 9: The relationship between special radii and internal friction angle.

4.5. Stress Level. The minimum confining pressure represents the stress level of the laneway surrounding rock. Figure 10 shows the relationship between the special radii and the stress level (minimum confining pressure). In Figure 10, the rock mechanics parameters are set as $C=3 \mathrm{MPa}$ and $\varphi=25^{\circ}$, the laneway radium is set as $2.5 \mathrm{~m}$, and the confining pressure ratio is set as $1.4(\eta=1.4)$. The characteristic radius has a positive correlation with the stress level, that is, with the increase of the stress level, the range of the plastic area will also increase. Under certain other conditions, the two curves are almost parallel, and the difference between the major axis radius and the minor axis radium does not change. It can be concluded that the stress

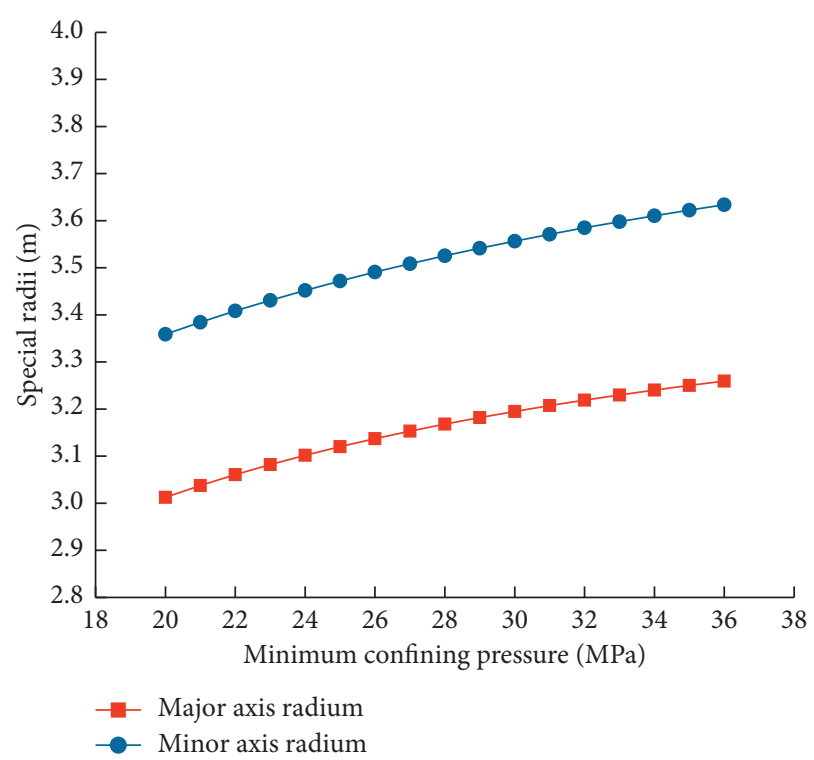

FIgURE 10: The relationship between special radii and stress level.

level is significant in the range of the plastic area but has little effect on the shape.

\section{Analysis of Applicable Conditions}

In the above analysis about comparison with existing theoretical formulas, when the confining pressure ratio is greater than 1.6, the deviation gradually increases with the increase of the confining pressure ratio. The error of boundary radius of plastic area calculated by the elliptic boundary equation is too large, and it is no longer applicable. The confining pressure ratio is the key factor affecting the shape of the laneway plastic area. To explore the reasons for the increase in error, we continue to study the evolution of plastic area morphology with confining pressure ratio. At this time, the variation range of confining pressure ratio is expanded, as shown in Figures 11 and 12. In Figures 11 and 12 , the rock cohesion is set as $5 \mathrm{MPa}$, the rock internal friction angle is set as $25^{\circ}$, the laneway radium is set as $2.5 \mathrm{~m}$, and the minimum confining pressure is set as the fixed value $\left(P_{3}=20 \mathrm{MPa}\right)$.

With the increase of confining pressure ratio, the shape of plastic area changes from ellipse to butterfly shape, as shown in Figure 11. When the confining pressure ratio is less than 1.6, both the maximum radius and the minimum radius of the plastic area lie on the coordinate axis, and the plastic area around the circular laneway is elliptical. When the confining pressure ratio is greater than 1.6, the minimum radius of the plastic area is on the coordinate axis, while the maximum radius of the plastic area is near the angle bisector of the four quadrants. And the plastic area of the circular laneway surrounding rock is butterfly shaped.

With the increase of the confining pressure ratio, the shapes of the plastic area change and the radii at different positions also have different changing rules, as shown in Figure 12. When the confining pressure ratio is less than 1.6, 


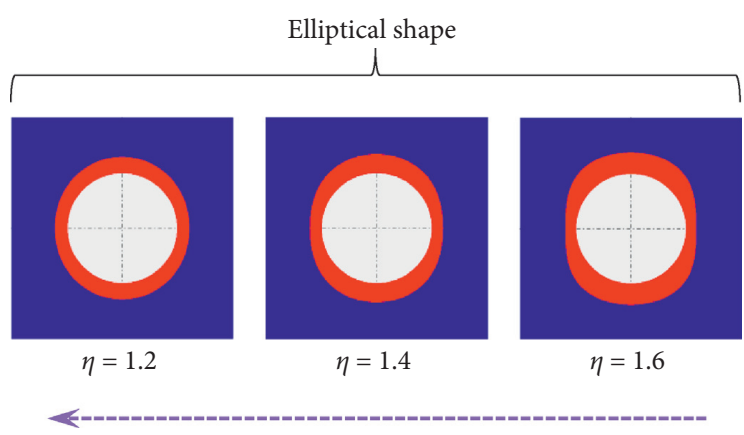

(a)

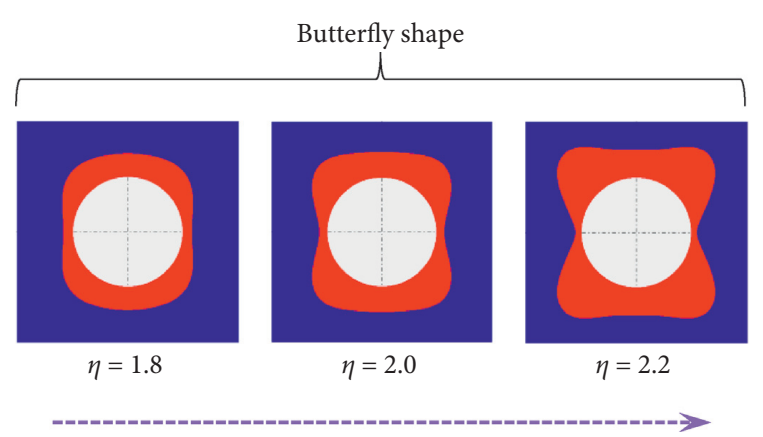

(b)

Figure 11: Evolution of the plastic area shape with confining pressure ratio $\left(a=2.5 \mathrm{~m} ; C=5 \mathrm{MPa} ; \varphi=25^{\circ} ; P_{3}=20 \mathrm{MPa}\right)$. (a) Elliptical shape. (b) Butterfly shape.

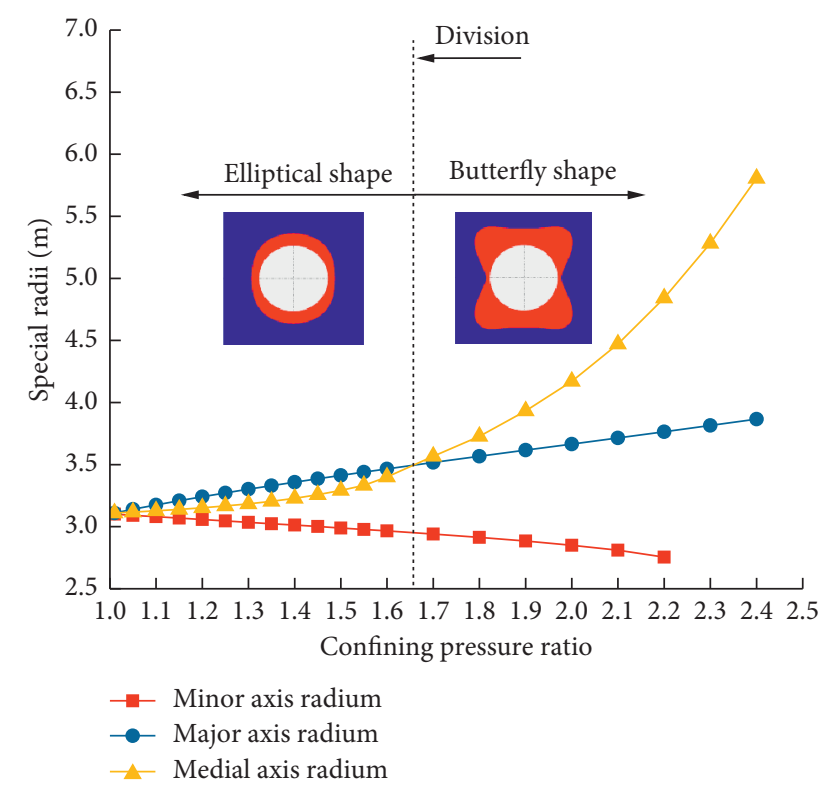

FIGURE 12: Radius variation curves at different positions and the division between plastic area shapes.

the medial axis radium is less than the major axis radium, and the plastic area of the circular laneway surrounding rock is elliptical. When the confining pressure ratio is greater than 1.6, the medial axis radium is larger than the major axis radium and the plastic area is butterfly shaped. The error of the boundary radius of the plastic area calculated by the elliptic boundary equation is large, and it is no longer applicable.

So, when the confining pressure ratio is less than 1.6, the plastic area of the circular laneway surrounding rock is elliptical, and the elliptic boundary equation is applicable. When the confining pressure ratio is greater than 1.6, the plastic area is butterfly shaped, and the elliptic boundary equation is no longer applicable.

\section{Conclusions}

This paper studied the evolution law of the plastic area shapes of the circular laneway surrounding rock from circular to elliptical and derived the analytical solutions of the boundary radii in the elliptical shape. Based on the work presented in this paper, the following conclusions are made:

(1) The shape evolution of the elliptical plastic area in nonuniform stress field was revealed. With the increase of the confining pressure ratio from 1 , the major axis radius of the plastic area increases gradually, the minor axis radius decreases gradually, and the shape of the plastic area gradually evolves from circular to elliptical.

(2) The analytical expressions of major axis and minor axis radii of the elliptical plastic area were derived, and the elliptic boundary equation of the plastic area of circular laneway in nonuniform stress was established. The analytical formula of the boundary radius at any position in the elliptic plastic area was obtained.

(3) The confining pressure ratio is the key factor affecting the shape of plastic area. When the confining pressure ratio is less than 1.6, the plastic area of the circular laneway surrounding rock is elliptical, and the elliptic boundary equation is applicable. When the confining pressure ratio is greater than 1.6, the plastic area is butterfly shaped, and the elliptic boundary equation is no longer applicable.

\section{Data Availability}

The data used to support the findings of this study are available from the corresponding author upon request.

\section{Conflicts of Interest}

The authors declare that they have no conflicts of interest.

\section{Acknowledgments}

This study was partially supported by the National Natural Science Foundation of China (grant nos. 52004289 and 51234006). 


\section{References}

[1] D. Lamich, M. Marschalko, I. Yilmaz et al., "Subsidence measurements in roads and implementation in land use plan optimisation in areas affected by deep coal mining," Environmental Earth Sciences, vol. 75, pp. 69-79, 2016.

[2] G. C. Zhang, Z. J. Wen, S. J. Liang et al., "Ground response of a gob-side entry in a longwall panel extracting $17 \mathrm{~m}$-thick coal seam: a case study," Rock Mechanics and Rock Engineering, vol. 53, no. 2, pp. 497-516, 2019.

[3] P. Wen, K. Soucek, L. Stas, and R. Singh, "Long-hole destress blasting for rockburst control during deep underground coal mining," International Journal of Rock Mechanics and Mining Sciences, vol. 61, pp. 141-153, 2013.

[4] D. Palumbo, R. Finis, F. Ancona et al., "Damage monitoring in fracture mechanics by evaluation of the heat dissipated in the cyclic plastic area ahead of the crack tip with thermal measurements," Engineering Fracture Mechanics, vol. 181, pp. 65-76, 2017.

[5] G. Zhang, L. Chen, Z. Wen et al., "Squeezing failure behavior of roof-coal masses in a gob-side entry driven under unstable overlying strata," Energy Science \& Engineering, vol. 8, no. 7, pp. 2443-2456, 2020.

[6] K. H. Lee, D. H. Kai, J. J. Park et al., "A study on critical strain based damage-controlled test for the evaluation of rock laneway stability," Journal of Medical Screening, vol. 13, pp. 91-97, 2011.

[7] H. Kastner, Statik des tunel und stollenbauess, Springer, Berlin, Germany, 1962.

[8] M. Qian, P. Shi, and J. Xu, Mining Pressure and Strata Control, China University of Mining and Technology Press, Xuzhou, China, 2010.

[9] M. Abdel-Meguid, R. K. Rowe, and K. Y. Lo, "Three-dimensional analysis of unlined tunnels in rock subjected to high horizontal stress," Canadian Geotechnical Journal, vol. 40, no. 6, pp. 1208-1224, 2003.

[10] H. Kang, X. Zhang, L. Si et al., "In-situ stress measurements and stress distribution characteristics in underground coal mines in China," Engineering Geology, vol. 116, pp. 333-345, 2010.

[11] M. J. Leitman and P. Villaggio, "Plastic zone around circular holes," Journal of Engineering Mechanics, vol. 135, no. 12, pp. 1467-1471, 2009.

[12] X. Yu, Y. Zheng, and H. Liu, Stability Analysis of Surrounding Rock in Underground Engineering, Coal Industry Press, Beijing, China, 1983.

[13] L. Chen, J. Peng, W. Fan et al., "Analysis based on unified strength theory of non-uniform stress field circular laneway for plastic area," Journal of China Coal Society, vol. 32, pp. 20-23, 2007.

[14] Z. Zhao, Study on mechanism and control method of deformation and failure of surrounding rock in large deformation mining laneway, $\mathrm{PhD}$ thesis, China University of Mining and Technology, Beijing, China, 2014.

[15] N. Ma and Z. Z. Li, "Distribution of the deviatoric stress field and plastic area in circular laneway surrounding rock," Journal of China University of Mining \& Technology, vol. 44, pp. 206-213, 2015.

[16] X. Guo, Z. Zhao, X. Gao, Z. Ma, and N. Ma, "The criteria of underground rock structure failure and its implication on rockburst in roadway: a numerical method," Shock and Vibration, vol. 2019, Article ID 7509690, 12 pages, 2019.

[17] X. Guo, N. Ma, X. Zhao, Z. Zhao et al., "The general shapes and criterion for surrounding rock mass plastic area of round laneway," Journal of China Coal Society, vol. 41, pp. 18711877, 2016.

[18] X. Guo, Z. Zhao, X. Gao, X. Wu, and N. Ma, "Analytical solutions for characteristic radii of circular roadway surrounding rock plastic zone and their application," International Journal of Mining Science and Technology, vol. 29, no. 2, pp. 263-272, 2019.

[19] A. E. Green, "The elastic equilibrium of isotropic plates and cylinders," Proceedings of the Royal Society of London, vol. 195, p. $533,1949$.

[20] H. G. Poulos and E. H. Davis, Elastic Solutions for Soil and Rock Mechanics, Wiley, New York, NY, USA, 1974.

[21] N. Ma, X. Zhao, Z. Zhao, X. Guo et al., "Conjecture of butterfly shape coal and gas outburst mechanism in excavation laneway," Journal of Mining Science and Technology, vol. 2, pp. 136-148, 2017.

[22] Z. Zhao, N. Ma, X. Guo et al., "Mechanism conjecture of butterfly rock burst in coal seam laneway," Journal of China Coal Society, pp. 2689-2697, 2016.

[23] Li Ji, X. Qiang, W. Wang et al., "Distribution law of principal stress difference of deep surrounding rock of gob-side entry and optimum design of coal pillar width," Tehnički Vjesnik, vol. 26, no. 6, pp. 1743-1752, 2019.

[24] J. Li, "The coal pillar design method for a deep mining laneway based on the shape of the plastic area in surrounding rocks," Arabian Journal of Geosciences, vol. 13, p. 454, 2020.

[25] Z. Zhao, N. Ma, X. Guo et al., "Falling principle and support design of butterfly-failure roof in large deformation mining laneways," Journal of China Coal Society, pp. 2932-2939, 2016.

[26] N. Ma, J. Li, X. Zhao et al., "High quality gas channel and its construction method applied to coal and gas simultaneous extraction in deep mining," Journal of China Coal Society, vol. 40, pp. 742-748, 2015. 